\title{
Advanced Image Processing Techniques for Extracting Regions of Interest using Multimode IR Processing
}

\author{
John. T. Caulfield ${ }^{\text {a }}$ Member, IEEE \\ Joseph P. Havlicek ${ }^{\mathrm{b}}$, Senior Member, IEEE \\ ${ }^{a}$ Cyan Systems, Santa Barbara, CA 93105 \\ ${ }^{b}$ Electrical and Computer Engineering Department, The University of Oklahoma, Norman, OK 73019
}

\begin{abstract}
As large format single color and multicolor sensors proliferate challenges in viewing and taking action on a much higher data volume becomes challenging to end users. We report on processing techniques to effectively extract targets utilizing multiple processing modes - both multiple spectral band and multiple pre-processed data bands from Focal Plane Array (FPA) sensors. We have developed image processing techniques which address many of the key pre-processing requirements, including scene based non-uniformity correction of static and dynamic pixels, multiband processing for object detection, and reduction and management of clutter and non-targets in a cluttered environment. Key motivations for these techniques include image pre-processing extracting small percentages of the image set with potentially high likelihood targets and then transmitting "active" pixel data while ignoring unchanging pixels. These techniques have demonstrated significant reductions in the raw data, and allow the end user to more intelligently select potential data types for object identification without requiring a person in the loop.
\end{abstract}

\section{INTRODUCTION}

Visible and IR sensors with increased resolution are being developed for improved optical detection in seeker and surveillance systems [1,2]. However, the high data rate associated with these sensors is continuing to place demands on the downstream image and signal processing electronics. Modern IR sensors technologies have advanced to the point where larger format sensors are creating so much data that recording and viewing of the data requires a larger and expensive computer and networking systems dedicated to the collection and interpretation of IR data. Although memory and storage technology costs and bandwidth are scaling at about the same rate as sensor formats, data transmission issues and non standard sensor types have shown a lack of continuity between sensor data collection and dissemination. This problem can have the result of bottlenecks, overwhelming the users of visible and IR imagery so that even cataloging data becomes computer and data storage intensive. This current research focuses on automated image cueing on to support proliferation of larger format sensors with smaller package sizes, ease of use, and more on-sensor functionality. By performing the appropriate multiple spectral mode signals of interest can be separated from noise and artifacts such as blinking pixels, image persistence, and fixed pattern noise in the FPA instead of the artifact being carried far down the signal chain, consuming bandwidth and other network resources to be corrected later in the external electronics.

Our goals is that on and near FPA pre-processing will be a practical solution to the data bottleneck challenges that plague the integration of very high resolution sensors onto small mobile unattended and man-portable based sensors.

\section{Multiband SEnSORS For Multimode PROCESSING}

Research and development continues in the field of multiband sensors, particularly for military applications. This work evaluates the benefits of multispectral and multiple mode imaging for object recognition in commercial applications. Cyan purchased a dual-band, $320 \times 256$ focal plane array from Qmagiq Inc. The detector consists of two QWIP structures stacked on top of one another, designed to absorb at peak wavelengths of $\sim 5 \mu \mathrm{m} \mathrm{MWIR} \mathrm{and} \sim 8.6 \mu \mathrm{m}$ LWIR bands [3], respectively. We purchased an SEIR electronics test set with programmable clocks and biases, and a pour fill dewar. We also purchased a 50-mm, F/2.3, NINOX wideband lens made by Janos Technology for data collection. The sensor head and electronics are shown in figure 1.

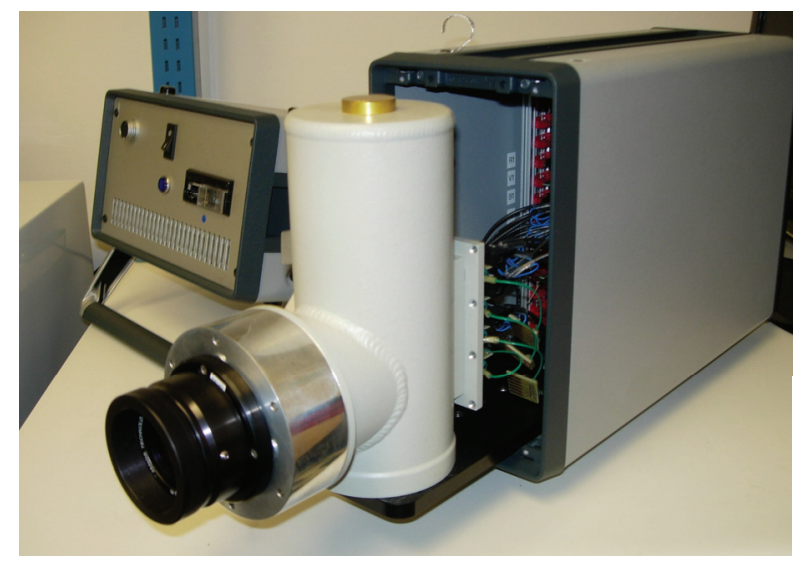

Figure 1. Dual Band Sensor head and Drive Electronics. 
Using a dual band sensor for detection, we can chose bands based on the quality of the imagery. Many factors affecting detection can be evaluated with a dual band sensor. Depending on object signature, location, time of day, atmospherics, detection and tracking in one band can be preferred over the other [4]. Figure 2 below shows imagery from the dual band FPA with the MWIR band on the left, and LWIR band on the right of the image.

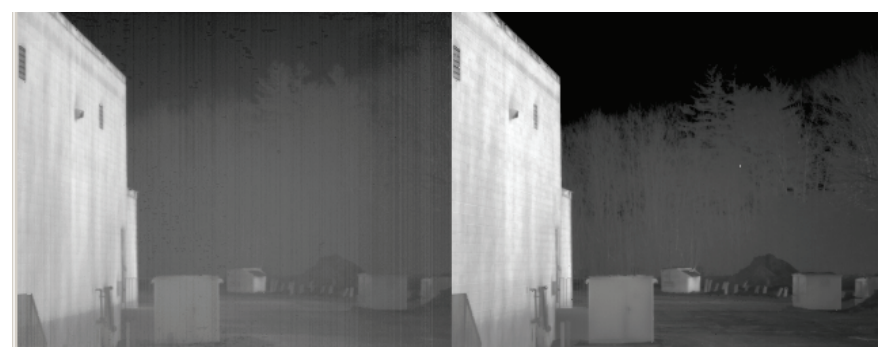

Figure 2. Outdoor imagery of Cyan's dual band IRFPA.

\section{IMAgE PRE-PROCESSING FOR TARGET CUEING}

Image Preprocessing and other related image processing transformations are being implemented in a number of different FPA technologies. Sensor designers are conducting research into implementing biologically inspired parallel processing in the ROIC to solve a number of important issues with detection, noise, and the need for more compact systems. Advanced imagers can be augmented with pre-processing that occurs near the sensor. This pre-processing provides the ability to pre-cue a target, track, and alert the user of potential threats autonomously. Sensors utilizing pre-processing are capable of autonomous use in surveillance, acquisition, and track scenarios. Figure 3 illustrates how biological vision systems perform temporal/spatial filtering and analog target cueing in the retina, and is motivated by a similar signal flow description posed by Gupta [5] and Knopf.

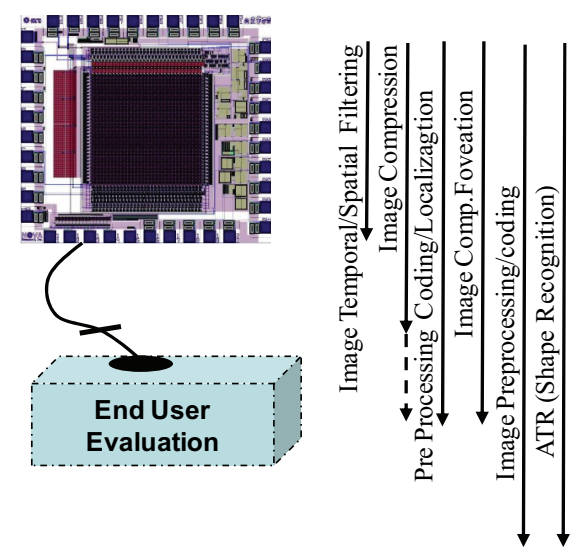

Figure 3. Architecture for automated region of interest cueing inspired by biological sensors.

For image temporal and spatial filtering, we have techniques that evaluate local changes in amplitude over a specified period of time, referred to as a temporal aperture. Other discriminants used in our developmental algorithm include: 1) Automated selection of best atmospheric transmission; 2) Rule based target detection; 3) Non common mode optical flow; 4) Evaluation of common band-band transients in ROI; 5) Common mode slowly varying intensity. An example of techniques using a waveform from a multimode temporal aperture to discriminate signal from noise is shown in figure 4.

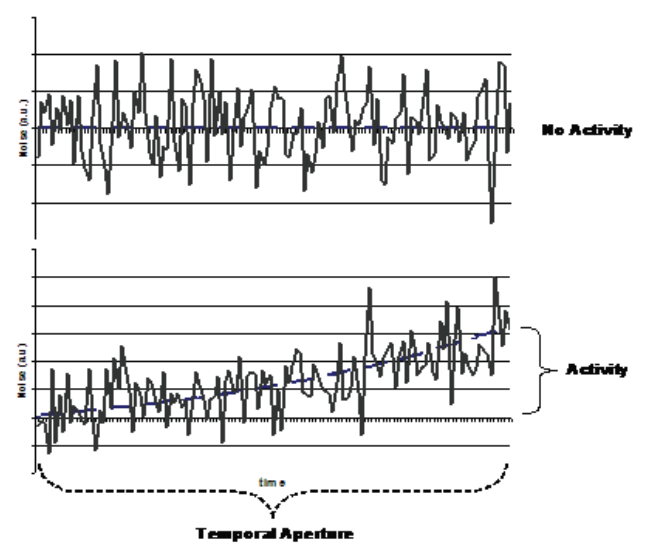

Figure 4. Adaptive change detection extracts potential target activity from surrounding detector field.

Key pre-processing requirements are for sensing amplitude and/or motion in either band. Pre-processing allow potential identification of objects of interest, and selectable moving targets in the field of regard. The activity sensing architecture is designed to inhibit or reject non useful information, such as benign background, static objects, sun glints, rural and urban clutter. Figure 5 illustrates how the pre-processing performed significantly reduces bandwidth.

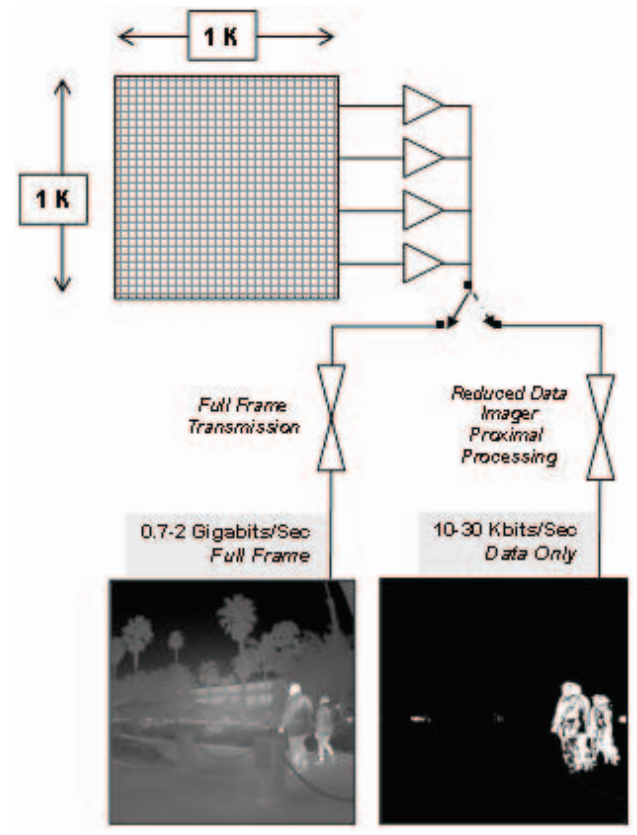

Figure 5. Network bandwidth requirements of full frame and reduced bandwidth pre- processed data. 
Continued work in near and on FPA processing should facilitate development of more advanced cognitive sensing paradigms. The long term goal of the activity sensor is to be able to process and extract extended targets, textural differences, and allow fully automated cognitive on-FPA routines comparable to today's spatial convolution and digital based ATR. When the multimode pre-processing is successful, we typically see target data extracted and other surrounding data suppressed.

\section{Processed Results}

We participated in testing at the local Santa Barbara airport measuring takeoff and landings of commercial aircraft. We took several data sets of jet and propeller aircraft. Several data sets lasted more than 3 minutes and were tracked for several miles after takeoff. We observed dynamically changing signatures when evaluating the data through the preprocessing algorithms. Modulation of the signature occurred in part due to atmospheric turbulence, and also the angle of the aircraft with respect to the sensor. We hypothesize that the MWIR signature was dimmer because of the exhaust gas dispersion, and the increased LWIR heating of engine components at take off, but this involves signature phenomenology analysis that goes beyond this papers scope. What was interesting is that signature changes were captured in both the MW and LW bands during takeoff. Figure 6 shows pre-processed data with only the aircraft in both bands after multimodal processing.

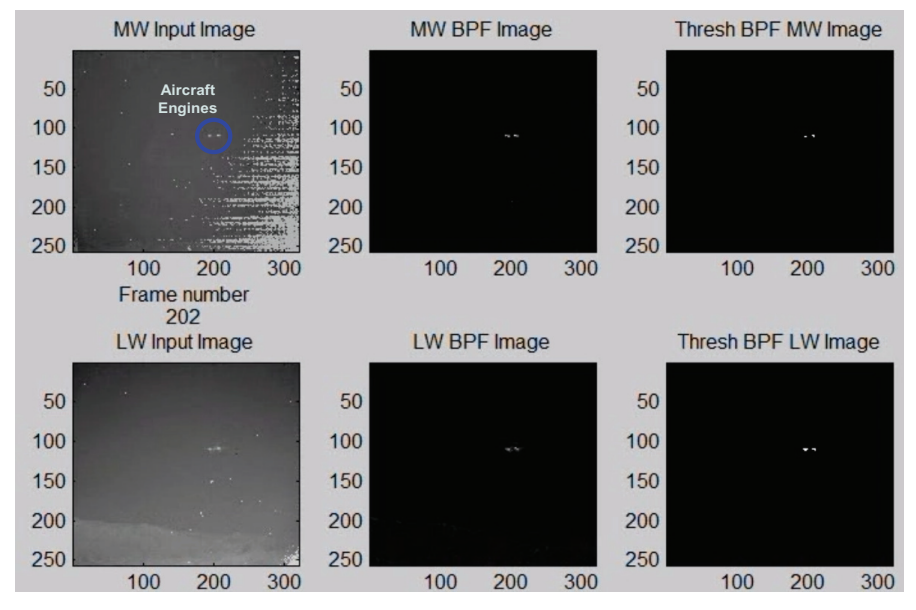

Figure 6. Detection of a small commercial jet aircraft in both MW and LW infrared bands.

\section{Summary}

We have developed multimode processing algorithms using different spectral bands and tunable bandpass filtering. These types of pre processing appear suitable for automated target detection and cueing. Our metric for success in the activity sensing development is to be able to detect targets of interest in a variety of backgrounds and clutter, without an increase in false alarms. We have attempted to compare this data to a highly optimized set of algorithms implemented in a downstream image processor. We have limited our algorithm simulation to data sets from a stationary camera for this initial research. In the near future we plan to work towards moving cameras/platforms and target in heavier clutter.

We believe that the multiple modes are applicable for many military, homeland security and commercial security applications. On-FPA processing is a necessary and natural trend in future sensor developments. The smaller features and the inclusion of mixed analog and digital signal components in the pixel have made this possible. Development of adaptive spectral processing is a potentially important technique for insuring detection in inclement weather and varying optical propagation conditions.

The long term vision is that near and multimode preprocessing will provide a great degree of automation and efficiency in the detection of different scenarios. Large format sensors will be able to retain their resolution and have a very high internal frame rate with a much slower external data rate, on the order of $<100 \mathrm{KHz}$. This lower bandwidth will enable wireless networks for interconnection of sensors, and making interfacing multipoint wireless sensor transmitters easier and more affordable.

The evolution of automated pre-processing can hopefully be accelerated with the understanding of biologically inspired sensory information processing. Figure 7 illustrates a roadmap of processing that is current performed in biological vision that we hope to exploit and mimic in machine vision to improve all aspects of sensing even up to the level of associated memory and cognitive recognition. 


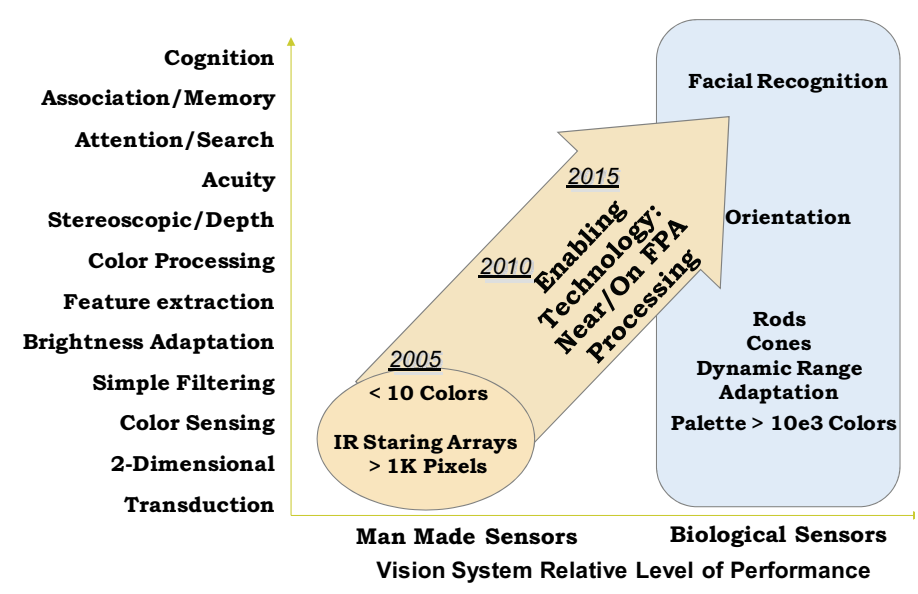

Figure 7. Roadmap of multiple layers of improved image processing based on understanding and adapting biological inspired architectures.

Advanced processing techniques may hold the key to managing and fully utilizing the benefits of large area, multiband and multifunctional infrared sensors.

\section{REFERENCES}

[1] J. T. Caulfield, et. al., "Advanced IRFPAs for next generation sensors", Proc. SPIE, vol. 5406, pp 178-183, 2004.

[2] P. Norton et. al., "Third-generation infrared imagers", Proc. SPIE vol. 4130, pp. 226-236, 2000.

[3] J. Bundas, et. al. "Absolute temperature measurements using a two color QWIP focal plane array", Proc. SPIE, Volume 7660, 2010.

[4] M. C. Dudzik "The Infrared \& Electro-Optical Systems Handbook", Vol. 4, pp. 245-298 1993

[5] M. M. Gupta and G. K. Knopf, Neuro-Vision Systems: Principles and Applications, 1995. 